\title{
Preocupações de docentes de Educação Física: associação com o nível de ensino e o vínculo institucional
}

Renato Daniel Trusz ${ }^{\mathrm{a} *} \oplus$, Jorge Both ${ }^{\mathrm{b}} \odot$, José Henrique dos Santos ${ }^{\mathrm{c}} \odot$, Alexandra Folle ${ }^{\mathrm{d}} \oplus$, Juarez Vieira do Nascimento ${ }^{\mathrm{e}} \odot$, Gelcemar Oliveira Farias ${ }^{\mathrm{d}} \odot$

\author{
Palavras Chave: \\ Trabalhadores; \\ Educação Física e \\ Treinamento; \\ Ensino Fundamental \\ e Médio; \\ Educação Infantil.
}

\begin{abstract}
RESUMO
O objetivo do estudo foi analisar as preocupações de professores de Educação Física considerando o nível de ensino e o vínculo empregatício. Participaram do estudo 61 professores de Educação Física de Balneário Camboriú, SC. Na coleta de dados foi utilizado um questionário sociodemográfico e a Escala de Preocupações de Professores. Os resultados indicaram que os professores eram muito preocupados com a tarefa e o impacto e observou-se associação significativa da dimensão consigo com o nível de ensino, enquanto que em relação ao vínculo empregatício dos professores investigados constatou-se associação significativa com a dimensão tarefa. Conclui-se que os diferentes níveis de atuação e o tipo de vínculo empregatício podem interferir nas inquietações associadas aos docentes.
\end{abstract}

\begin{abstract}
The aim of this study was to analyze the concerns of physical education teachers considering the teaching level and the employment relationship. Sixty-one Physical Education teachers from Balneário Camboriú, SC, participated in this study. In data collection, a sociodemographic questionnaire and the Teacher Concerns Scale were used. The results indicated that teachers were highly concerned about the task and the impact and significant association of the self dimension and the teaching level was observed, whereas regarding the employment relationship of investigated teachers, significant association with the task dimension was found. It could be concluded that the different levels of action and the employment relationship may interfere with concerns associated with teachers.
\end{abstract}

\section{RESUMEN}

El objetivo del estudio fue analizar las preocupaciones de profesores de Educación Física considerando el nivel de enseñanza y el vínculo laboral. Participaron del estudio 61 profesores de Educación Física de Balneario Camboriú, SC. Para la recolección de datos se usó un cuestionario sociodemográfico y la Escala de Preocupaciones de Profesores. Los resultados mostraron que los profesores se preocupan por la tarea y el impacto y se observó una asociación significativa entre dimensión consigo y el nivel de enseñanza, mientras que con relación al vínculo laboral de los profesores investigados se constató una asociación significativa con la dimensión tarea. Se concluye que los diferentes niveles de actuación y el tipo de vínculo laboral pueden interferir en las inquietudes de los docentes.

\footnotetext{
a Prefeitura Municipal de Balneário Camboriú, Balneário Camboriú, Santa Catarina, Brasil.

b Universidade Estadual de Londrina, Londrina, Paraná, Brasil.

' Universidade Federal Rural do Rio de Janeiro, Seropédica, Rio de Janeiro, Brasil.

dUniversidade do Estado de Santa Catarina, Florianópolis, Santa Catarina, Brasil.

e Universidade Federal de Santa Catarina, Florianópolis, Santa Catarina, Brasil.
}

\author{
*Autor correspondente: \\ Renato Daniel Trusz \\ E-mail: renato.pedagogicobc@yahoo.com.br
}




\section{INTRODUÇÃO}

Apesar da incipiência de estudos sobre a temática na área de Educação Física, ressalta-se que o suporte teórico que tem orientado as investigações é respaldado pelos estudos de Fuller (1969, p. 220-221) e Fuller e Bown (1975, p. 37), nos quais se concebe três estágios de preocupações do professor ao longo de sua carreira, quais sejam: consigo, tarefa e impacto. As preocupações consigo são típicas de professores iniciantes e estão associadas à insegurança em assumir o papel de professor, ao ser avaliado, bem como a necessidade de ter o controle disciplinar dos alunos.

As preocupações com a tarefa estão associadas ao número excessivo de alunos, a quantidade e a disponibilidade de materiais, ao espaço físico adequado, a carga horária e a continuidade do planejamento. Por fim, surgem as preocupações com o impacto, associadas ao atendimento das necessidades individuais dos alunos, a identificação de problemas de aprendizagem, ao desafio de discentes desmotivados e a orientação para sua formação integral. A experiência como docente contribui para que as preocupações apresentem caráter evolutivo, mudando de uma perspectiva eminentemente pessoal para ações que denotam preocupações com o sucesso escolar dos alunos (FULLER, 1969; FULLER; BOWN, 1975).

Asinvestigaçõessobre as preocupações de professores buscam identificar as dimensões predominantes em estudantes na situação de estágio (MARSO; PIGGE, 1994; BEJARANO; CARVALHO, 2004; PIOVANI; BOTH; NASCIMENTO, 2012; RIBEIRO et al., 2015) e em professores em serviço (CONKLE, 1996; FARIAS, 2000; FOLLE; NASCIMENTO, 2011; COSTA, 2013). Além disso, procuram compreender os fatores intervenientes na carreira dos professores na escola (GONÇALVES, 2009) e no ensino superior (RALPH, 2004), no intuito de melhor explicar o impacto das preocupações no contexto da intervenção profissional.

Neste cenário, as preocupações de professores de Educação Física que atuam em níveis distintos de ensino necessitam ser aprofundadas (CONKLE, 1996). Diferente dos demais níveis de ensino, a educação infantil é recente na legislação e nos currículos escolares em nível nacional (BARBOSA, 2010), o que demonstra a necessidade da realização de investigações a respeito da inserção e das preocupações do professor de Educação Física nesse nível de ensino (MELLO et al., 2014; COSTA; PEDRINI; KUNZ, 2014). Docentes dos demais níveis podem apresentar preocupações diferenciadas, pois as características dos alunos e a dimensão das escolas entre promovem situações que causam descontentamentos, angústias e desmotivações nos professores, pois investigações com este intuito incrementam a produção do conhecimento sobre a prática pedagógica docente relacionada à cultura de movimento adequada à infância (GARANHANI, 2005).

Neste cenário, um fator que pode distinguir as preocupações de professores diz respeito ao vínculo empregatício nas redes de ensino. Enquanto professores efetivos, que estão aprovados em seus estágios probatórios, podem sentir-se mais seguros e autônomos na profissão, professores admitidos em caráter temporário (ACTs) estão sujeitos a experimentar insegurança diante da instabilidade do vínculo e da possibilidade de transferências, sendoIhe frequentemente exigida a reinserção em novas unidades escolares ao início de cada semestre ou ano letivo (CONCEIÇÃO et al., 2015). Afinal, a experiência, a competência e/ou a expertise pedagógicas são influenciadas pelo contexto de ensino (BERLINER, 2004), assim como pela socialização ocupacional docente, pelos contextos e culturas escolares (FREITAS, 2011).

A ocorrência destas situações pode favorecer a manifestação de preocupações diferenciadas em professores efetivos e ACTs, assim como em docentes que atuam em distintos níveis de ensino da educação básica. Considerando os tempos, os espaços, as condições de trabalho e a especificidades de cada nível e do vínculo empregatício dos professores, o objetivo do estudo foi analisar as preocupações de professores de Educação Física, considerando o nível de ensino (educação infantil e ensino fundamental) e a natureza do vínculo empregatício (ACT e efetivo).

\section{MÉTODO}

A investigação caracteriza-se como um estudo descritivo, de corte transversal com abordagem quantitativa dos dados, o qual foi aprovado pelo Comitê de Ética em Pesquisa com Seres Humanos de uma universidade pública do Brasil (1.345.476/2015). Cabe destacar que todos os participantes assinaram o Termo de Consentimento Livre Esclarecido antes de iniciar a participação na investigação.

Do universo de 74 professores de Educação Física atuantes na rede municipal de Educação da cidade de Balneário Camboriú, estado de Santa Catarina, participaram da investigação 61 professores $(82,4 \%$ da população), sendo 27 atuantes na educação infantil, 34 no ensino fundamental, 37 ACT e 24 efetivos.

$\mathrm{Na}$ coleta de dados foi empregado um questionário composto pelos dados sociodemográfico e pelo Teacher Concerns Questionnaire - Physical Education - TCQPE (MCBRIDE, 1993). Os dados sociodemográficos se reportaram ao sexo, data de nascimento, experiência como atleta, preferência por faixa etária para trabalhar, formação, tempo de experiência, atuação e vínculo, 
- qual foi traduzido e validado semanticamente para a realidade brasileira por Costa (2013), como Escala de Preocupações de Professores. A Escala de Preocupações de Professores apresenta 15 assertivas relacionadas à cada uma das três dimensões (consigo, tarefa, impacto) propostas no modelo teórico, em uma escala ordinal de um a cinco (não preocupado, pouco preocupado, preocupado, muito preocupado e extremamente preocupado).

$\mathrm{Na}$ análise das questões referentes às preocupações dos professores foi utilizada, inicialmente, a equação de ponderação apresentada por Farias et al. (2008, p. 312). A partir da inserção das equações no programa Microsoft Excel foi possível quantificar o nível de preocupação em cada uma das três dimensões. O resultado da equação representa um índice percentual com 200 pontos de variabilidade de $-100,0 \% a+100,0 \%$.

A partir da determinação dos índices relativos às preocupações dos professores nas três dimensões, foram estabelecidos pontos de corte com base na escala de variabilidade da equação de ponderação para a definição dos níveis de preocupação, assim concebidos: Baixa preocupação: - 100,0\% a -20,0\%; Preocupação moderada: - 19,9\% a +19,9\%; e Alta preocupação: $+20,0 \%$ a $+100,0 \%$ (FARIAS et al., 2008). Entretanto, para possibilitar a análise dos dados foram agrupadas as categorias "baixa" e "moderada" preocupação. Assim, os professores foram categorizados em pouco $(-00,0 \%$ a $+19,9 \%)$ ou muito $(+20,0 \%$ a $+100,0 \%)$ preocupado.

$\mathrm{Na}$ análise estatística a qual buscou avaliar a associação entre as preocupações e as variáveis nível de atuação e vínculo empregatício, empregou-se o teste Qui-quadrado, sendo adotado o nível de significância de $95 \%(p \leq 0,05)$. Os testes estatísticos foram realizados no software SPSS, versão 21).

\section{RESULTADOS}

A maioria dos professores investigados eram mulheres $(67,2 \%)$, possuía curso de especialização $(73,7 \%)$, atuava no ensino fundamental $(55,7 \%)$, era ACT $(60,6 \%)$, trabalhava 40 horas semanais $(83,6 \%)$ e não atuava em outra rede de ensino (83,6\%) (Tabela 1$)$.

$\mathrm{Em}$ relação às preocupações dos professores (Tabela 2), observou-se que os docentes eram muito preocupados com as dimensões tarefa $(72,1 \%)$ e impacto $(85,2 \%)$. Embora a maioria dos professores tenha se apresentado pouco preocupada com os aspectos associados à dimensão consigo $(65,6 \%)$.

Ao avaliar as preocupações dos professores, considerando os níveis de ensino (Tabela 3), observouse associação significativa apenas na avaliação da dimensão consigo $(p<0,01)$, sendo que a maioria dos professores que atuava na educação infantil $(85,2 \%)$ era
Tabela 1. Características sociodemográficas dos professores de Educação Física de Balneário Camboriú.

\begin{tabular}{lc}
\hline Variáveis sociodemográficas & $\mathbf{n}(\%)$ \\
\hline Sexo & \\
Feminino & $41(67,2 \%)$ \\
Masculino & $20(32,8 \%)$ \\
Formação & \\
Licenciatura & $14(23,0 \%)$ \\
Especialização & $45(73,7 \%)$ \\
Mestrado & $2(3,3 \%)$ \\
Nível de Atuação & \\
Ed. infantil & $27(44,3 \%)$ \\
Ens. fundamental & $34(55,7 \%)$ \\
Vínculo empregatício & \\
ACT & $37(60,6 \%)$ \\
Efetivo & $24(39,4 \%)$ \\
Carga Horária & \\
20 horas & $6(9,9 \%)$ \\
30 horas & $4(6,5 \%)$ \\
40 horas & $51(83,6 \%)$ \\
Vínculo com outra rede de ensino & \\
Sim & $10(16,4 \%)$ \\
Não & $51(83,6 \%)$ \\
\hline
\end{tabular}

Fonte: Os autores (2018).

Tabela 2. Nível de preocupações dos professores de Educação Física Balneário Camboriú.

\begin{tabular}{lcc}
\hline \multirow{2}{*}{ Preocupações } & \multicolumn{2}{c}{ Nível de Preocupações } \\
\cline { 2 - 3 } & Pouco n(\%) & Muito n(\%) \\
\hline Consigo & $40(65,6)$ & $21(34,4)$ \\
Tarefa & $17(27,9)$ & $44(72,1)$ \\
Impacto & $9(14,8)$ & $52(85,2)$ \\
\hline
\end{tabular}

Fonte: Os autores (2018).

pouco preocupada, enquanto no ensino fundamental, observou-se que metade dos docentes apresentava muita preocupação $(50,0 \%)$.

Ao considerar a natureza do vínculo empregatício dos professores investigados (Tabela 4), constatou-se que apenas a dimensão tarefa evidenciou associação significativa $(p=0,05)$, sendo que o professores ACT $(81,1 \%)$ eram mais preocupados com a tarefa de ensinar do que os docentes efetivos (58,3\%).

\section{DISCUSSÃO}

As preocupações com o impacto foram elevadas entre os docentes atuantes na educação infantil e no ensino fundamental, sendo que resultados semelhantes foram 
Tabela 3. Nível de preocupações dos professores considerando o nível de ensino.

\begin{tabular}{lccc}
\hline \multirow{2}{*}{ Nível de Ensino } & \multicolumn{2}{c}{ Nível de Preocupações } & \multirow{2}{*}{ P } \\
\cline { 2 - 3 } & Pouco n(\%) & Muito n(\%) & \\
\hline Consigo & & & \\
Ens. fundamental & $17(50,0 \%)$ & $17(50,0 \%)$ & $<0,01^{*}$ \\
Ed. infantil & $23(85,2 \%)$ & $4(14,8 \%)$ & \\
Tarefa & & & \\
Ens. fundamental & $7(20,6 \%)$ & $27(79,4 \%)$ & 0,16 \\
Ed. infantil & $10(37,0 \%)$ & $17(63,0 \%)$ & \\
Impacto & & & \\
Ens. fundamental & $4(11,8 \%)$ & $30(88,2 \%)$ & 0,46 \\
Ed. infantil & $5(18,5 \%)$ & $22(81,5 \%)$ & \\
\hline
\end{tabular}

Fonte: Os autores (2018).

Tabela 4. Nível de preocupações considerando o vínculo empregatício.

\begin{tabular}{|c|c|c|c|}
\hline \multirow{2}{*}{$\begin{array}{l}\text { Vínculo } \\
\text { Empregatício }\end{array}$} & \multicolumn{2}{|c|}{ Nível de Preocupações } & \multirow{2}{*}{$\mathbf{p}$} \\
\hline & Pouco n(\%) & Muito n(\%) & \\
\hline \multicolumn{4}{|l|}{ Consigo } \\
\hline Efetivo & $16(66,7 \%)$ & $8(33,3 \%)$ & \multirow{2}{*}{0,89} \\
\hline ACT & $24(64,9 \%)$ & $13(35,1 \%)$ & \\
\hline \multicolumn{4}{|l|}{ Tarefa } \\
\hline Efetivo & $10(41,7 \%)$ & $14(58,3 \%)$ & \multirow{2}{*}{$0,05^{*}$} \\
\hline ACT & $7(18,9 \%)$ & $30(81,1 \%)$ & \\
\hline \multicolumn{4}{|l|}{ Impacto } \\
\hline Efetivo & $2(8,3 \%)$ & $22(91,7 \%)$ & \multirow{2}{*}{0,26} \\
\hline ACT & $7(18,9 \%)$ & $30(81,1 \%)$ & \\
\hline
\end{tabular}

Fonte: Os autores (2018).

apresentados em outros estudos (CONKLE, 1996; FOLLE; NASCIMENTO, 2011; COSTA, 2013; VIEIRA; BEUTTEM MULLER; BOTH, 2018), que também verificaram que as preocupações com o impacto se destacam das demais em reação aos professores da educação básica investigados. Ao mesmo tempo em que os achados deste estudo são similares as pesquisas envolvendo professores de Educação Física (PIOVANI; BOTH; NASCIMENTO, 2012; VIEIRA; BEUTTEM MULLER; BOTH, 2018).

Embora na literatura as preocupações consigo sejam evidenciadas nos primeiros anos da docência, é perceptível o equilíbrio de indivíduos nas categorias pouco e muito preocupado entre os que atuam no ensino fundamental, diferente dos que atuam na educação infantil que se apresentam menos preocupados do que preocupados em relação a estas questões. Destaca-se que as preocupações associadas à dimensão consigo configuram-se como aquelas sentidas durante as primeiras experiências com o ensino, como a necessidade de sentir-se bem como professor e a assunção de responsabilidades concernentes (FARIAS; NASCIMENTO, 2012), de ser aceito pelos pares, ter seu trabalho reconhecido e manter controle dos alunos. As preocupações são centradas na própria imagem, performance e adequação (BEJARANO; CARVALHO, 2004), as quais são preocupações típicas do choque inicial com a realidade da escola (FARIAS; NASCIMENTO, 2012).

Uma das explicações para a baixa preocupação consigo nos professores atuantes na educação infantil diz respeito à inserção desse nível de ensino ser muito recente no âmbito da educação, incorporada somente em 1996 por meio da Lei de Diretrizes e Bases da Educação Nacional (LDB) (BRASIL, 2015), ao passo que o município em que foi realizada a pesquisa a incorporou somente no ano de 2006 (BALNEÁRIO COMBURIU, 2013). Fruto da sua incorporação à educação básica, a educação infantil tem sidocriticamente discutidaa partir de distintas concepções que refletem, entre outros, a indissociabilidade entre o educar e cuidar (BARBOSA, 2010), o currículo e a formação profissional para atuar nesta etapa de ensino (LACERDA; COSTA, 2012).

Apesar das diferentes ciências e vertentes teóricas que embasam a formação do professor de Educação Física, ainda existe crítica quanto a falta de adequação dos currículos e dos projetos pedagógicos em formar docentes efetivamente preparados para atuar na educação infantil (SAYÃO, 2002), na perspectiva de superar o descompasso entre as necessidades e os desejos da criança e a intencionalidade pedagógica dos professores (SAYÃO, 2002).

Esta constatação, articulada à ausência de um currículo explícito estruturado e à atitude favorável das crianças face à Educação Física, permite interpretar que a baixa preocupação dos professores consigo pode estar associada à ausência de um referencial didáticopedagógico fundada em uma concepção de ensino (ainda que inconsciente) vinculada à promoção de diversão, pois os deixaria mais confortáveis na função docente, por convergir para uma concepção comum entre os entes escolares, cuja visão sobre a Educação Física figura exclusivamente na perspectiva do lúdico e do recreativo.

Todavia, para outros profissionais da unidade é possível que a diversidade de concepções da disciplina dificulte as comparações e as avaliações acerca do trabalho dos professores de Educação Física, visto que a prática pedagógica na educação infantil historicamente se constituiu, tendo por base diversas influências teóricas (OLIVEIRA, 2005; COSTA; PEDRINI; KUNZ, 2014) e minimizando, assim, as preocupações consigo desses professores. 
No ensino fundamental, por sua vez, é possível que as preocupações consigo tenham origem em uma complexa rede de fatores relativos ao processo formal de escolarização, no qual interagem a explicitação de um currículo estruturado e a exigência em sistematizáIo (BOSCATTO; DARIDO, 2017). De modo similar, dos fatores relacionados ao controle dos processos educativos pelos gestores escolares, além do status da disciplina (e do professor) na escola frente as outras áreas disciplinares e gestores educacionais (LOYOLA; FONTE; FIGUEIREDO, 2010; FREITAS, 2012), o amadurecimento dos alunos e a consequente expressão de atitudes mais autônomas frente aos conteúdos e às diretrizes assumidas pelos professores (GUIMARÃES et al, 2001; OLIVEIRA; DAOLIO, 2014).

$O$ estabelecimento de critérios para selecionar e organizar o currículo no ensino fundamental pode acarretar significativa preocupação para os professores, por um lado, por ocorrer em meio a distintas concepções, abordagens e perspectivas que caracterizam a Educação Física e, por outro, não se constituir exclusivamente em ato pedagógico, mas, também com reflexos nas dimensões política e administrativa natural do ambiente escolar (BOSCATTO; DARIDO, 2017). Esta condição pode representar preocupação para os professores, em ter suas práticas controladas e/ou avaliadas por gestores e pares escolares que nem sempre conhecem as múltiplas expressões da disciplina, levando-os a colocar em causa o sentimento de capacidade para a docência.

No plano do desenvolvimento curricular, a confrontação da intenção de ensino do professor com os interesses e necessidades dos alunos repercute no temor de não conseguir gerir de forma adequada o processo ensino aprendizagem. $O$ nível de maturidade dos alunos, principalmente na segunda fase do ensino fundamental, leva-os a questionar o conteúdo, as diretrizes fornecidas pelo professor, até se autoexcluírem das aulas (OLIVEIRA; DAOLIO, 2014), o que pode traduzir em comportamentos de indisciplina(SANT'ANA;NASCIMENTO;AZEVEDO, 2015) que fragilizam o professor no que diz respeito ao seu papel e autoridade frente aos alunos, acarretando insegurança em proporcionar um ambiente produtivoà aprendizagem.

Da mesma forma, no ensino fundamental também foi apresentado o maior número de professores preocupados com as dimensões impacto e tarefa do que aqueles que atuam na educação infantil, dado que pode ser justificado por questões intrínsecas ao ensino e a faixa etária dos alunos nesse nível. Em relação à dimensão tarefa, o trabalho com turmas muito grandes e divisão de materiais e espaço podem ser fatores que propiciem a maior frequência dessas preocupações. A preocupação quanto ao sucesso escolar dos alunos no ensino fundamental, pelas exigências sistemáticas e cultura assimilada como a tradicional na fase inicial de escolarização, diferentemente e infelizmente do que ainda se concebe na educação infantil, pode ser um fator associado a dimensão impacto que exerce forte influência nas preocupações desses professores.

Considerando a natureza das preocupações apresentadas por Fuller (1969, p. 220-221) e Fuller e Bown $(1975$, p. 37), compreende-se o predomínio das preocupações com o impacto entre os professores da educação infantil e do ensino fundamental. Este dado é evidente na literatura, como no estudo de Farias (2000, p. 51) que também identificou predomínio de impacto seguido da tarefa. Atender as necessidades de alunos, identificar dificuldades de aprendizagem, proporcionar seu desenvolvimento integral e individualizar o planejamento, configuram-se como inquietações decorrentes do contexto atual, em que muitos alunos se afastam da escola ou das aulas de Educação Física.

Estudos realizados em outras redes públicas de ensino (FARIAS, 2000; FOLLE; NASCIMENTO, 2011; COSTA, 2013) também encontraram resultados similares, o que revela a inquietação constante dos professores quanto às condições de trabalho que lhes são proporcionadas. A falta de condições apropriadas de trabalho, associadas ao material que é utilizado, ao espaço físico, ao tempo para planejamento, ao excesso de tarefas e de alunos são fatores que dificultam o trabalho do professor, fazendo com que os docentes se sintam impossibilitados de atingir seus objetivos junto aos estudantes.

É de supor que a semelhança do contexto de atuação de professores não distinga as preocupações consigo de efetivos e ACT, na medida em que não se observaram diferenças significantes entre estes professores, a partir da constatação da frequência de cerca de um terço para as duas categorias docentes. No entanto, apesar de ambas serem imputadas quase as mesmas responsabilidades funcionais, as preocupações com a tarefa distinguiram significativamente entre professores efetivos e ACT, com a maior quantidade de temporários apresentando preocupações com a tarefa.

Considerando-se que as escolas possuem condições estruturais e materiais similares, uma possível explicação para a maior preocupação evidenciada pelos professores ACT, na dimensão tarefa, pode estar atrelada ao regime funcional destes docentes e a consequente necessidade de recorrer a estratégias que visam resolver as restrições postas pela carência de materiais ou potencializá-los, bem como se munir didática e metodologicamente para lograr êxito na disciplina.

O conhecimento e a integração do professor junto à comunidade escolar por mais de um ano, beneficiado pelo vínculo efetivo, favorece o maior conhecimento dos alunos, identificando problemas e dificuldades 
específicos e consequentemente buscando atender e individualizar o ensino. Diferentemente, para os professores $\mathrm{ACT}$, o vínculo com a comunidade escolar e a continuidade do trabalho tornam-se mais dificultosos, em vista do encerramento do contrato a cada ano escolar, fato que pode comprometer não só o trabalho docente, mas a educação em si, visto que esta precisa de segurança e continuidade para atingir seus fins (NAUROSKI, 2014).

Os estudos que abordam as condições de trabalho de professores ACT (NAUROSKI, 2014) têm apontado a precariedade de condições a que estes professores são submetidos, visto que muitos não têm direito à escolha de unidade educacional ou turmas em decorrência da classificação no processo seletivo, restando geralmente as escolas e turmas que não são do agrado dos professores efetivos (CONCEIÇÃO; FRASSON; BOROWSKI, 2014). Assim, acabam por trabalhar com os recursos materiais $\mathrm{e}$ físicos que sobram, além de, por vezes, terem seu planejamento e trabalho interrompidos quando do retorno de um professor efetivo à vaga.

Ao tratar dos dados sobre o vínculo empregatício dos professores, em relação à dimensão impacto, pode-se perceber que os resultados deste estudo são semelhantes aos referenciados na literatura, envolvendo as preocupações de professores em serviço (CONKLE, 1996; FARIAS, 2000; FOLLE; NASCIMENTO. 2011; COSTA, 2013), os quais indicam esta dimensão de preocupações como a predominante. Os estudos relacionados à temática das preocupações de professores em serviço (CONKLE, 1996; FARIAS, 2000; FOLLE; NASCIMENTO, 2011; COSTA, 2013), mesmo alguns relatando a presença de professores ACT em suas amostras, não foram realizadas comparações, considerando os vínculos empregatícios. A diferença encontrada entre professores de Educação Física ACT e efetivos em relação à dimensão tarefa, sugere que o tipo de vínculo exerce influência nas preocupações, o que revela a necessidade de identificação e compreensão de fatores relacionados à vida profissional destes profissionais no âmbito das unidades educacionais. Ainda assim, apesar das condições distintas de vínculos institucionais, observou-se como positivo a preservação do comprometimento dos professores de ambas as categorias com o sentido educacional de sua ação, visto que a maior proporção dos investigados demonstrou preocupação com o impacto.

\section{CONCLUSÕES}

Ao final deste estudo, pode-se concluir que os resultados se alinham aos encontrados na literatura, em que as preocupações relacionadas ao impacto são predominantes em professores em serviço. Ao averiguar as preocupações em relação ao nível de ensino em que os professores atuam, constatou-se similaridade entre as preocupações no que diz respeito às dimensões impacto e tarefa. Quanto à dimensão consigo, evidenciou-se que os professores atuantes na educação infantil preocupam-se menos do que seus pares atuantes no ensino fundamental.

A análise das preocupações condicionadas ao tipo de vínculo empregatício dos professores de Educação Física com a rede pública municipal de ensino identificou que os professores ACT são mais preocupados com questões relacionadas à dimensão tarefa e, apesar de não disporem das condições que julgam adequadas para o desenvolvimento da tarefa educativa, se esmeram, demonstrando o quanto se preocupam com a visibilidade de seu trabalho, vislumbrando a possibilidade de manutenção de vínculos futuros.

Destaca-se que uma das limitações do estudo se refere a realidade investigada, pois, devido as características próprias do contexto, os resultados não podem ser generalizados, visto que se configuram como específicos da realidade da rede pública municipal de ensino investigada. Assim, no âmbito disciplinar, foi um estudo realizado com professores de Educação Física, que apresentam especificidade quanto a sua prática pedagógica no contexto escolar.

A partir das reflexões apresentadas, vislumbra-se a necessidade de realizar novos estudos com o intuito de esclarecer como os fatores intrínsecos aos distintos níveis de ensino, como questões pedagógicas e estruturais, implicam na caracterização das preocupações dos professores. Por fim, conclui-se que aspectos relacionados ao contexto de atuação dos professores devem ser levados em consideração em futuras investigações relacionadas à temática, visto que tanto o nível de escolaridade quanto o vínculo empregatício apresentaram-se como fatores intervenientes na manifestação de preocupações nos professores. Sugerese, ainda, a realização de estudos em outras realidades e com a utilização de outros instrumentos, de forma que a compreensão acerca das preocupações de professores em serviço possa ser ampliada.

\section{REFERÊNCIAS}

Balneário Camboriú. Orientações Curriculares Municipais de Balneário Camboriú - Educação Infantil. Blumenau: Nova Letra, 2013.

Barbosa MC. Especificidades da ação pedagógica com os bebês. In: Anais do I Seminário Nacional: Currículo em Movimento - perspectivas atuais; 2010 nov; Belo Horizonte, Brasil. Belo Horizonte: p. 1-17.

Bejarano NRR, Carvalho AMP. A História de Ely: um professor de Física no início da carreira. Revista Brasileira de Ensino de Física 2004; 26(2): 165-178. 
Berlinder DC. Expert teachers: Their characteristics, development and accomplishments. In: Batllori I, Obiols R, Gomez Martinez $\mathrm{AE}$, Oller I, Freixa M, Pages I, Blanch J, Ed. De la teoria... a l'aula: Formacio del professorat ensenyament de las ciències socials. Barcelona, Spain: Departament de Didàctica de la Llengua de la Literatura I de les Ciències Socials, Universitat Autònoma de Barcelona; 2004. p. 13-28.

Boscatto JD, Darido SC. Currículo e educação física escolar: análise do estado da arte em periódicos nacionais. J. Phys. Educ 2017; 28: e2855.

Brasil. Lei no. 9394, de 20 de dezembro de 1996. Lei de Diretrizes e Bases da Educação Nacional. 11. Edições Câmara, 2015.

Conceição VJS, Frasson JS, Medeiros CR, Wittizorecki ES, Krug HN. A organização escolar e o trabalho docente de professores iniciantes de Educação Física de Criciúma - SC. Pensar a Prática 2015; 18(4): 769-781.

Conceição VJS, Frasson JS, Borowski EBv. A influência da socialização sobre o percurso docente dos professores de Educação Física no início da carreira. Pensar a Prática 2014 jan/mar; 17(2): 472-484.

Conkle T. Inservice physical educators' stages of concerns: A test of fuller's model and the TCQ-PE. Physical Educator 1996; 3(53): 122-131.

Costa AR, Pedrini L, Kunz E. O "brincar e se movimentar" como fundamento básico da Educação Física na educação infantil. In: Pereira BO, Silva AN, Cunha AC, Nascimento JV, organizadores. Atividade Física, Saúde e Lazer: olhar e pensar o corpo. Florianópolis: Tribo da llha; 2014. p. 89-103.

Costa BO. Preocupações pedagógicas e desenvolvimento profissional em Educação Física: passo ou descompasso? Seropédica. Dissertação [Mestrado em Educação] - Instituto de Educação/Instituto Multidisciplinar da Universidade Federal Rural do Rio de Janeiro; 2013.

Farias GO, Folle A, Both J, Saad M, Teixeira A, Salles W, Nascimento JV. Preocupações pedagógicas de estudantesestagiários na formação inicial em Educação Física. Motriz $2008 \mathrm{jul} / \mathrm{set}$; 14(3): 310-319.

Farias GO. O percurso profissional dos professores de Educação Física: rumo à prática pedagógica. Florianópolis. Dissertação [Mestrado em Educação Física] - Centro de Desportos da Universidade Federal de Santa Catarina; 2000.

Farias GO, Nascimento JV. Construção da identidade profissional: metamorfoses na carreira docente em educação física. In: Farias GO, Nascimento JV, organizadores. Construção da identidade profissional em educação física: da formação à intervenção. Florianópolis: Ed. Da UDESC; 2012. p. 61-80.

Folle A, Nascimento JV. Preocupações ao longo da carreira docente: estudos de caso com professores de educação física do magistério público estadual. Revista Brasileira de Ciências do Esporte 2011 out/dez; 33(4): 841-856.

Freitas RC. Estudo multicasos sobre a socialização profissional de professores de Educação Física em início de carreira. Seropédica. Dissertação [Mestrado em Educação]. Instituto de Educação / Instituto Multidisciplinar da Universidade Federal Rural do Rio de Janeiro, 2011.

Fuller F. Concerns of teachers: a developmental conceptualization. American Education Research Journal 1969 mar; 6(2): 207-226.

Fuller F, Bown O. Becoming a teacher. In: Rehage KJ, Editor. Teacher Education: The seventy-fourth Yearbook of the National Society for the Study of Education. Chicago: University of Chicago Press; 1975. p. 25-52.
Garanhani MC. Os saberes de educadoras da pequena infância sobre o movimento do corpo infantil. In: Anais do Congresso Brasileiro de Ciências do Esporte e Congresso Internacional de Ciências do Esporte; 2005; Porto Alegre, Brasil. Porto Alegre: CBCE, 2005.

Gonçalves HAPR. A Motivação para a Carreira Docente: contributos para o Estudo das principais preocupações em início de carreira. Faro. Dissertação [Mestrado em Supervisão] - Faculdade de Ciências Humanas e Sociais da Universidade do Algarve, 2009.

Guimarães AA, Pellini FC, Araujo JSR, Mazzini JM. Educação física escolar: atitudes e valores. Revista Motriz 2001; 7: 17-22.

Lacerda CG, Costa AMB. Educação Física na Educação Infantil e o Currículo da Formação Inicial. Rev. Bras. Ciênc. Esporte $2012 \mathrm{abr} / \mathrm{jun} ; 34(2)$ : 327-341.

Loyola RC, Della Fonte SS, Figueiredo ZCC. Experiências profissionais e os sentidos atribuídos à educação física em contexto escolar. Movimento 2010 jan/mar; 17(1):177-193.

Marso RN, Pigge FL. Outstanding teachers' concerns about teaching at four stages of career development. [paper presented at Annual Conference of the Association of Teachers Educators; 1994 feb 12-16; Atlanta, Estados Unidos].

McBride RE. The TCQ-PE: An adaptation of the Teacher Concerns Questionnaire Instrument to a physical education setting. Journal of Teaching in Physical Education 1993; 12: 188-196.

Mello AS, Santos W, Klippel MV, Rosa AP, Votre SJ. Educação Física na educação infantil: produção de saberes no cotidiano escolar. Revista Brasileira de Ciências do Esporte 2014 abr/jun; 36(2): 467-484.

Nauroski EA. Trabalho docente e subjetividade: a condição dos professores temporários (PSS) no Paraná. Curitiba. Tese [Doutorado em Sociologia] - Setor de Ciências Humanas da Universidade Federal do Paraná; 2014.

Oliveira NRC. Concepção de Infância na Educação Física brasileira: primeiras aproximações*. Revista Brasileira de Ciências do Esporte 2005; 26(3): 95-109.

Oliveria RC, Daolio J. Educação física, prática pedagógica e nãodiretividade: a produção de uma "periferia da quadra". Educação em Revista 2014; 30(02): 71-94.

Piovani VGS, Both J, Nascimento JV. Preocupaciones pedagógicas de los Estudiantes practicantes de Educación Física de diferentes domicilios sociales de Uruguay. Movimento, 2012 out/dez; 18(04): 77-98.

Ralph EG. Interns' and cooperating teachers' concerns dining the extended practicum. The Alberta Journal of Educational Research 2004; 50(4): 411-429.

Ribeiro VT, Folle A, Farias GO, Nazário PF. Preocupações pedagógicas e competência profissional de estudantes de Educação Física em situação de estágio. Revista da Educação Física/UEM 2015 jan/mar; 26(1): 59-68.

Sant'ana ASS, Nascimento JV, Azevedo ES. Estratégias Pedagógicas no trato com a indisciplina na Educação Física escolar. In: Nascimento JV, Souza ERS, Ramos V, Rocha JCS, organizadores. Educação Física e esporte: Convergindo para novos caminhos... Florianópolis: Ed. Da UDESC; 2015. p. 271-292.

Sayão D. Corpo e movimento: notas para problematizar algumas questões relacionadas à educação infantil e à Educação Física. Revista Brasileira de Ciências do Esporte 2002; 23(2): 55-68.

Vieira SV, Beuttemmuller LJ, Both J. Preocupações de professores de Educação Física conforme os ciclos de desenvolvimento profissional e características sociodemográficas. Journal of Physical Education 2018; 29(e2924): 1-9. 